\title{
Chromosome evolution in fishes: a new challenging proposal from Neotropical species
}

\author{
Mauro Nirchio ${ }^{1}$, Anna Rita Rossi ${ }^{2}$, Fausto Foresti ${ }^{3}$ and Claudio Oliveira ${ }^{3}$
}

We present a database containing cytogenetic data of Neotropical actinopterygian fishes from Venezuela obtained in a single laboratory for the first time. The results of this study include 103 species belonging to 74 genera assigned to 45 families and 17 out of the 40 teleost orders. In the group of marine fishes, the modal diploid number was $2 n=48$ represented in $60 \%$ of the studied species, while in the freshwater fish group the modal diploid complement was $2 \mathrm{n}=54$, represented in $21.21 \%$ of the studied species. The average number of chromosomes and the mean FN were statistically higher in freshwater fish than in marine fish. The degree of diversification and karyotype variation was also higher in freshwater fish in contrast to a more conserved cytogenetic pattern in marine fish. In contrast to the assumption according to which 48 acrocentric chromosomes was basal chromosome number in fish, data here presented show that there is an obvious trend towards the reduction of the diploid number of chromosomes from values near $2 n=60$ with high number of biarmed chromosomes in more basal species to $2 \mathrm{n}=48$ acrocentric elements in more derived Actinopterygii.

Se presenta una base de datos que contiene los datos citogenéticos de peces Actinopterigios Neotropicales de Venezuela obtenidos por primera vez en un solo laboratorio. Los resultados de este estudio incluyen 103 especies pertenecientes a 74 géneros de 45 familias contenidas en 17 de los 40 órdenes de teleósteos. En el grupo de peces marinos, el número diploide modal fue $2 \mathrm{n}=48$ representado en $60 \%$ de las especies estudiadas, mientras que en el grupo de peces de agua dulce el complemento diploide modal fue $2 \mathrm{n}=54$, representado en el $21,21 \%$ de las especies estudiadas. El número de cromosomas y FN promedio fueron estadísticamente superiores en peces dulceacuícolas. El grado de diversificación y variación en el cariotipo también fue mayor en peces de agua dulce en contraste con un patrón citogenético más conservado en peces marinos. En contraposición a la suposición según la cual 48 cromosomas acrocentricos era el número cromosómico basal en los peces, los datos aquí presentados muestran que existe una evidente tendencia hacia la reducción del número de cromosomas desde valores cercanos a $2 n=60$ con alto número de cromosomas birrámeos en las especies más basales a $2 n=48$ elementos acrocéntricos en los actinopterigios más derivados.

Key words: Actinopterygii, Ancestral karyotype, Diploid number, Fundamental number.

\section{Introduction}

Fishes represent more than half of all extant vertebrates with more than 32,000 recognized species (Eschmeyer et al., 2014), and are characterized by different morphology, behavior, and habitat (Nelson, 2006). The study of chromosomes in these animals has been expanding significantly thanks to the development of refined techniques of cell and tissue culture originally developed for mammals, but later adapted to the fish physiology (Clem et al., 1961; Booke, 1968; Wolf \& Quimby, 1969; Denton, 1973) and the development of less expensive in vivo direct methods (Osuf-Costaz \& Foresti, 1992). Because of the basal position that ray-finned fishes occupy in the phylogeny of vertebrates, studies on chromosomes of this group have provided valuable information for the understanding of different aspects such as mechanisms of sex determination and evolution of sex chromosomes, distribution of the nucleolus organizers regions (NOR), existence of supernumerary chromosomes and the role of polyploidy in evolution (Pisano et al., 2007).

Data on fish chromosomes are reported in a wide range of sources such as journals, proceedings and reports; however,

\footnotetext{
${ }^{1}$ Departamento de Acuacultura, Escuela de Ciencias Aplicadas del Mar, Universidad de Oriente, Porlamar, Isla de Margarita, Venezuela. mauro.nirchio@gmail.com

${ }^{2}$ Department of Biology and Biotechnology “C. Darwin”, Sapienza University of Rome, Italy. annarita.rossi@uniroma1.it ${ }^{3}$ Departamento de Morfologia, Instituto de Biociências, Universidade Estadual Paulista "Júlio de Mesquita Filho". 18618-970, Botucatu, SP, Brazil. fforesti@ibb.unesp.br, claudio@ibb.unesp.br
} 
these sources are often on local species not readily available for users, making it difficult for scientists or students to obtain general but detailed information on the cytogenetic characteristics of fishes, useful for comparative, evolutionary, and phylogenetic studies. Several checklists on cytogenetic data have been published (Denton, 1973; Park, 1974; Ojima et al., 1976; Vasiliev, 1985; Sola et al., 1981; Oliveira et al., 1988; Porto et al., 1992; Klinkhardt et al., 1995). The most recent one (Arai, 2011) cover about 3,425 species/subspecies of agnathans, cartilaginous fish, actinopterygians (ray-finned fish) and sarcopterygians (lobe-finned fish), which represent barely $10.7 \%$ of recognized species at global level. The main problem with the available checklists is that source data were obtained by a large number of different researchers, working with different methodologies (different antimitotic concentration and time of exposure) and with different standard as reference (different criteria of chromosome classification and fundamental number, FN, calculation), generating data that are difficult to compare. Indeed, it has been reported that chromosome size may be poorly evaluated due to the selection made by individual researcher, as observed in plants (Guerra, 2012). Furthermore, data for many important fish groups are still lacking in fish lists. Thus, although the general data are very important for our knowledge of fish cytogenetics, the analyses and interpretations on chromosome evolution in the ray finned fish are actually limited by the problems referred to above.

Venezuela is ranked in the top 10 countries with the greatest biodiversity on the planet, both in aquatic and terrestrial environments (Miloslavich, 2003). The presence of a shoreline that stretches along the Caribbean Sea and Atlantic Ocean (ca. 3,964 Km), which includes several distinct aquatic environments (sand, rocky littorals, seagrass meadows, coral reefs, communities of soft bottom, and mangrove forests), and tracts of continental water also with a variety of environmental conditions (rivers, coastal lagoons, marshes, lakes, and reservoirs), explains the great fish diversity. Fishbase registers at least 941 freshwater and 807 marine species in Venezuela (Froese \& Pauly, 2013).

In order to reach a better understanding of chromosome evolution in actinopterygian fishes, we karyotyped 103 Venezuelan species belonging to a wide range of evolutionary lineages (Table 1) with the same methodology and trying to cover a wide variety of freshwater and marine species.

\section{Material and Methods}

Fishes of 103 species of different orders were captured with diverse fishing methods (hook, snorkeling, cast nets, bag nets, seine nets, arrowhead fish-trap) and transported alive to laboratory where they were analyzed. Catalog numbers of the voucher specimens of species whose results are firstly presented here are given in Table 2 (LBP, Laboratório de Biologia e Genética de Peixes, UNESP, Botucatu, Brazil; ECAM, Escuela de Ciencias Aplicadas del Mar, Universidad de Oriente, Núcleo Nueva Esparta, Venezuela).
Table 1. Number of analyzed freshwater and marine Actinopterygii. Classification followed Eschmeyer \& Fong (2014).

\begin{tabular}{lccccc}
\hline \multicolumn{1}{c}{ Order } & Families & Genera & Species & Marine & Freshwater \\
\hline Anguilliformes & 1 & 1 & 2 & 2 & 0 \\
Aulopiformes & 1 & 1 & 1 & 1 & 0 \\
Batrachoidiformes & 1 & 4 & 4 & 4 & 0 \\
Beloniformes & 2 & 3 & 4 & 4 & 0 \\
Beryciformes & 1 & 1 & 1 & 1 & 0 \\
Characiformes & 5 & 12 & 18 & 0 & 18 \\
Cyprinodontiformes & 2 & 2 & 2 & 1 & 1 \\
Elopiformes & 1 & 1 & 1 & 1 & 0 \\
Gymnotiformes & 2 & 2 & 2 & 0 & 2 \\
Lophiiformes & 1 & 1 & 1 & 1 & 0 \\
Mugiliformes & 1 & 2 & 7 & 6 & 1 \\
Perciformes & 15 & 26 & 38 & 36 & 2 \\
Pleuronectiformes & 1 & 2 & 2 & 2 & 0 \\
Scorpaeniformes & 3 & 4 & 6 & 6 & 0 \\
Siluriformes & 4 & 8 & 9 & 2 & 7 \\
Synbranchiformes & 1 & 2 & 2 & 0 & 2 \\
Tetraodontiformes & 2 & 2 & 3 & 3 & 0 \\
\hline \multicolumn{1}{c}{ Total } & 44 & 75 & 103 & 70 & 33 \\
\hline
\end{tabular}

Mitotic chromosomes were obtained by injecting a solution of colchicine $0.0125 \%$ ( $1 \mathrm{cc} / 100 \mathrm{~g}$ of weight), exposure to the alkaloid for $50 \mathrm{~min}$, obtaining cell suspension from kidney tissue, hypotonic treatment for $20 \mathrm{~min}$ and centrifugation-fixation (3 times) with Carnoy's solution (Nirchio \& Oliveira, 2006). Special care was taken to standardize exposure to colchicine and hypotonic treatment times in order to achieve similar chromosomal condensation grades and to keep uniform criteria for comparisons.

Chromosomes in the karyotype were organized in a decreasing order of size and grouped by morphological types according to the ratio of lengths of their arms ( $\mathrm{r}=$ long arm length/short arm length) as metacentric ( $\mathrm{m}, \mathrm{r}=1.00$ to 1.70$)$, submetacentric (sm, $r=1.71$ to 3.00), subtelocentric (st, $r=$ 3.01 to 7.00) and acrocentric (a, r > 7.01) (Levan et al., 1964).

Data were analyzed with non-parametric test (Sokal \& Rohlf, 1995). The non-parametric variance analysis of KruskalWallis was used to verify the existence of statistical differences in the diploid number (2n) of chromosomes and in the FN between freshwater and marine fish. The association between the proportion of species with 48 exclusively acrocentric chromosomes and the environment (marine or freshwater), as well as the association between the number of species with $2 n=48$ acrocentric chromosomes among the Perciformes and among the other orders of Actinopterygii were tested using the Chi-squared test of independence.

Absence of normality in the data examined determined an inability to perform regression analysis. Hence, scatter plots and $95 \%$ concentration ellipses were created for the diploid number of chromosomes and FN (arm number) against the Nelson's (2006) phylogenetic position of Actinopterygii families in PAST (Hammer et al., 2001). Ellipses with 95\% confidence are used as a correlation indicator. When two variables are not correlated, the ellipse is circular in shape and it becomes more elongated when the correlation between two variables is stronger. To establish whether there was a correlation between these parameters and the phylogenetic 
position of the orders, a test of correlation by Spearman rank was conducted using WinSTAT.

Additionally, data on diploid number and DNA content per haploid nucleus (C-value) of fish species from which both values are available at the Animal Genome Size Database (Gregory, 2012) were used to establish the correlations between these parameters and phylogenetic position as following: a) for those data expressed in ranges, the average value of the range was calculated; b) multiple records were averaged to provide a single value for each species. The correlation analysis was performed first considering all the species and then excluding the possible polyploids. To standardize our data, we considered the distribution of the available $\mathrm{C}$-value data for non-polyploid ray-finned fish species reported by Smith \& Gregory (2009) that range from 0.35 to $4.20 \mathrm{pg}$., with a mean of $<1.1 \mathrm{pg}$, and with the vast majority of species falling within a smaller range of about 0.5 to $2.0 \mathrm{pg}$. Thus we assumed that $\mathrm{C}$-values $=1$ represents normal values for diploids; $\mathrm{C}$-values $<2$ would represent partial duplications of DNA sequences and C-values $<1$ represents partial DNA loss. Values $>2$ would represent polyploidy events.

\section{Results}

The results of this study include 103 species (70 marine and 33 freshwater) belonging to 74 genera ascribed to 45 families and 17 out of the 40 teleost orders (Table 1). Diploid number (2n), karyotype formula, and arm number (FN) are shown in Table 2. The diploid number ranged from $2 n=24$ in Mugil curema Valenciennes, 1836 (Mugilidae: Mugiliformes) to $2 \mathrm{n}=100$ in Potamorhina sp. (Curimatidae: Characiformes) and Prionotus punctatus (Bloch, 1793) (Triglidae:
Scorpaeniformes), with a mode of 48 chromosomes represented in 45 of the 103 studied species (43.7\%). FN ranged from 110 in Pygocentrus cariba (Humboldt, 1821), Serrasalmus irritans Peters, 1877 and S. rhombeus (Linnaeus, 1766) (Characiformes) to 33-34 in Stephanolepis setifer (Bennet, 1831) (Tetraodontiformes). In 26 out of 103 species (25.24\%) a karyotype composed of 48 exclusively acrocentric chromosomes $(2 \mathrm{n}=48, \mathrm{FN}=48)$ was observed. In the group of marine fishes, the modal diploid number was $2 n=48$ represented in $60 \%$ of the studied species, while in the freshwater fish group the modal diploid complement was $2 n=$ 54 , represented in $21.21 \%$ of the studied species. Both the number of chromosomes and FN were statistically higher in freshwater fishes than in marine fishes $\left(\mathrm{H}=32.730 ; P=1.06 \times 10^{-8}\right.$ and $\mathrm{H}=39.007 ; P=4.22 \times 10^{-10}$ respectively).

Statistical significant results were obtained also when numbers of chromosomes were compared with the species habitat (Table 3). Twenty-five out of 70 marine fish species (corresponding to $35.71 \%$ ) show 48 acrocentric chromosome, compared to a very low frequency (3.03\%) observed in freshwater fishes $\left(\chi^{2}=12.696 ; P \leq 0.0004\right)$. The relative proportions of species with 48 exclusively acrocentric chromosomes became even higher (57.89\%) and dependency stronger $\left(\chi^{2}=34.02 ; P \leq 0.0001\right)$ when the group of Perciformes was compared to the other orders of Actinopterygii.

The scatter plot of the diploid chromosome numbers (2n) and the arm number (FN) against Nelson's (2006) phylogenetic position for the Actinopterygii (Fig. 1) provides an overview of the relationship between those two variables. Spearman rank test revealed a significant inversely proportional relationship between phylogenetic position and diploid number $\left(\rho=-0,5126 ; P=1.55 \times 10^{-8}\right)$ and also between phylogenetic position and chromosome arm number $\left(\rho=-0.7106 ; P=2.0953 \times 10^{-17}\right)$.
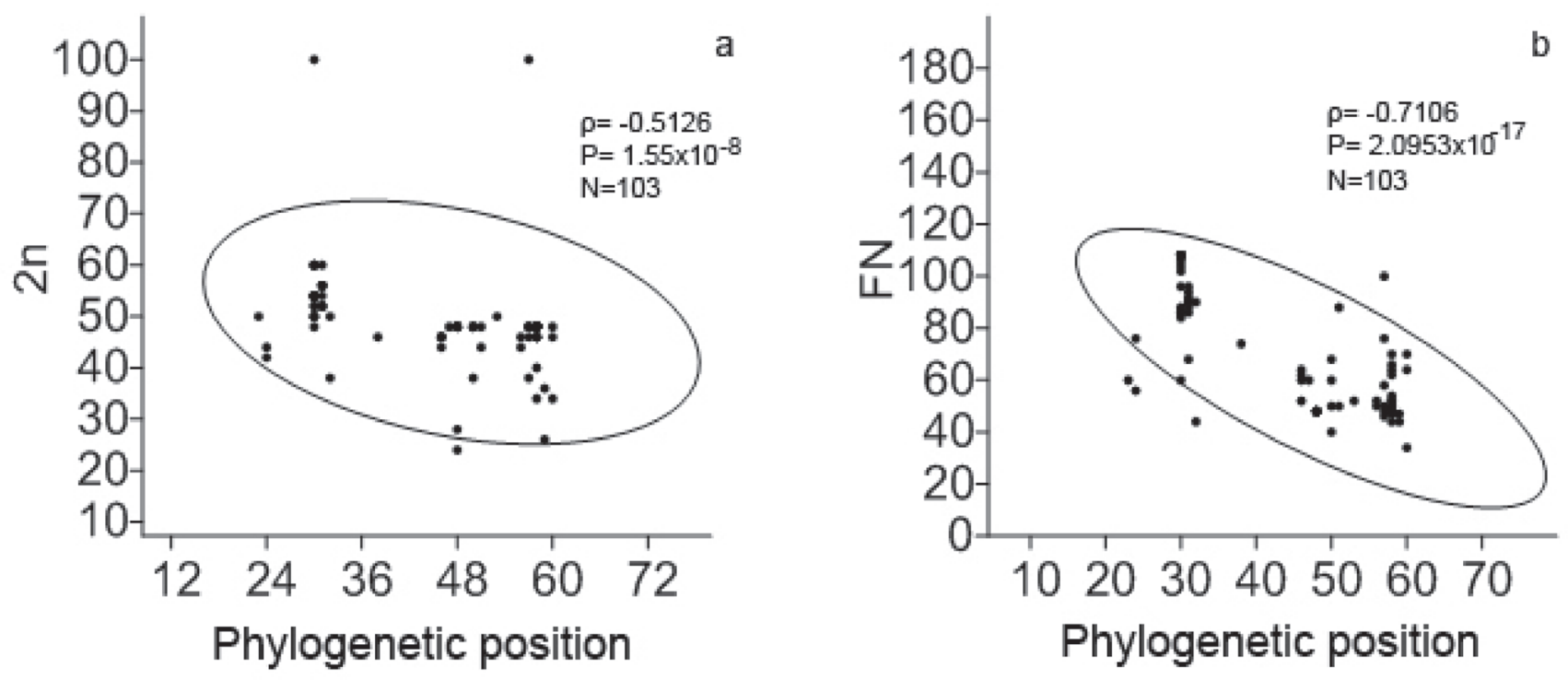

Fig. 1. Scatter-plot of (a) diploid number (2n) and (b) fundamental number (FN), against the phylogenetic position of Actinopterygii families presented by Nelson (2006) for 103 fish species. Ellipses with 95\% confidence are used as a correlation indicator. 
Table 2. Karyotype characteristics for 103 species of fishes from Venezuela. $\mathrm{M}=$ marine, $\mathrm{F}=$ freshwater, $\mathrm{m}=$ meta-, $\mathrm{sm}=$ submeta-, $\mathrm{st}=$ subtelo- and $\mathrm{a}=$ acrocentric chromosomes. In the last column the catalog numbers of the voucher specimens of species whose results are firstly presented here. Classification follows Eschmeyer \& Fong (2014).

\begin{tabular}{|c|c|c|c|c|c|c|c|}
\hline Order & Family & Species & Habitat & $2 n$ & Karyotype formulae & $\mathrm{FN}$ & $\begin{array}{l}\text { Reference or catalog } \\
\text { number of voucher }\end{array}$ \\
\hline \multirow[t]{2}{*}{ Anguilliformes } & Muraenidae & Gymnotorax moringa & $\mathrm{M}$ & 44 & $12 \mathrm{~m}+32 \mathrm{a}$ & 56 & ECAM 661 \\
\hline & & G. ocellatus & M & 42 & $34 \mathrm{~m} / \mathrm{sm}+8 \mathrm{a}$ & 76 & ECAM 682 \\
\hline Aulopiformes & Synodontidae & Synodus foetens & M & 46 & $28 \mathrm{~m} / \mathrm{sm}+18 \mathrm{a}$ & 74 & LBP 6095 \\
\hline \multirow[t]{4}{*}{ Batrachoidiformes } & Batrachoididae & Amphichthys cryptocentrus & M & 46 & $4 m+2 s m+40 a$ & 52 & Nirchio et al. 2001 \\
\hline & & Batrachoides manglae & M & 46 & $8 m+6 s m+32 a$ & 60 & Nirchio et al. 2001 \\
\hline & & Porichthys plectrodon & M & 44 & $8 \mathrm{~m}+10 \mathrm{sm}+6 \mathrm{st}+20 \mathrm{a}$ & 68 & Nirchio et al. 2004 b \\
\hline & & Thalassophryne maculosa & M & 46 & $12 m+6 s m+20 s t+8 a$ & 84 & Nirchio et al. $2004 \mathrm{a}$ \\
\hline \multirow[t]{4}{*}{ Beloniformes } & Belonidae & Strongylura marina & M & 48 & $2 \mathrm{~m}+46 \mathrm{a}$ & 50 & LBP 6077 \\
\hline & & S. timucu & M & 48 & $12 m+36 a$ & 60 & LBP 6078 \\
\hline & & Tylosurus crocodrilus & M & 48 & $8 m+12 s m+28 a$ & 68 & LBP 6100 \\
\hline & Hemiramphidae & $\begin{array}{l}\text { Hyporhamphus } \\
\text { unifasciatus }\end{array}$ & M & 38 & $2 \mathrm{~m}+36 \mathrm{a}$ & 40 & LBP 6099 \\
\hline \multirow[t]{3}{*}{ Beryciformes } & Holocentridae & Holocentrus adscensionis & M & 50 & $2 m+6 s t+42 a$ & 58 & LBP 6072 \\
\hline & Bryconidae & Brycon amazonicus & $\mathrm{F}$ & 50 & $22 \mathrm{~m}+14 \mathrm{sm}+14 \mathrm{st}$ & 100 & Mariguela et al. 2010 \\
\hline & & B. falcatus & $\mathrm{F}$ & 50 & $18 \mathrm{~m}+16 \mathrm{sm}+16 \mathrm{st}$ & 100 & LBP 7686 \\
\hline \multirow[t]{16}{*}{ Characiformes } & Characidae & Astyanax bimaculatus & $\mathrm{F}$ & 50 & $4 m+6 s m+28 s t+12 a$ & 88 & LBP 13292 \\
\hline & Curimatidae & Potamorhina altamazonica & $\mathrm{F}$ & 100 & $4 \mathrm{sm}+96 \mathrm{a}$ & 104 & LBP 3056 \\
\hline & Erythrinidae & $\begin{array}{l}\text { Hoplerythrinus } \\
\text { unitaeniatus }\end{array}$ & $\mathrm{F}$ & 48 & $32 \mathrm{~m}+16 \mathrm{sm}$ & 96 & ECAM 317 \\
\hline & Prochilodontidae & Prochilodus mariae & $\mathrm{F}$ & 54 & $40 \mathrm{~m}+14 \mathrm{sm}$ & 108 & Oliveira et al. 2003 \\
\hline & & Prochilodus reticulatus & $\mathrm{F}$ & 54 & $34 \mathrm{~m}+20 \mathrm{sm}$ & 108 & LBP 6127 \\
\hline & & Semaprochilodus kneri & $\mathrm{F}$ & 54 & $40 m+14 s m$ & 108 & Oliveira et al. 2003 \\
\hline & & S. laticeps & $\mathrm{F}$ & 54 & $40 \mathrm{~m}+14 \mathrm{sm}$ & 108 & Oliveira et al. 2003 \\
\hline & & Colossoma macropomum & $\mathrm{F}$ & 54 & $54 \mathrm{~m}$ & 108 & Nirchio et al. $2003 \mathrm{~b}$ \\
\hline & & Mylossoma duriventris & $\mathrm{F}$ & 54 & $54 m / 53 m+1 a$ & $108 / 107$ & LBP 7206 \\
\hline & & Piaractus brachypomus & $\mathrm{F}$ & 54 & $54 \mathrm{~m}$ & 108 & Nirchio et al. $2003 \mathrm{~b}$ \\
\hline & Serrasalmidae & Pygocentrus cariba & $\mathrm{F}$ & 60 & $18 \mathrm{~m}+30 \mathrm{sm}+2 \mathrm{st}+10 \mathrm{a}$ & 110 & Gaviria et al. 2005 \\
\hline & & Serrasalmus altuvei & $\mathrm{F}$ & 60 & $26 \mathrm{~m}+22 \mathrm{st}+12 \mathrm{a}$ & 108 & ECAM 1015 \\
\hline & & S. irritans & $\mathrm{F}$ & 60 & $22 \mathrm{~m}+20 \mathrm{sm}+8 \mathrm{st}+10 \mathrm{a}$ & 110 & ECAM 997 \\
\hline & & S. rhombeus & $\mathrm{F}$ & 60 & $30 m+16 s m+4 s t+10 a$ & 110 & Nirchio et al. 2002 \\
\hline & Triportheidae & Triportheus orinocensis & $\mathrm{F}$ & 52 & $20 m+14 s m+12 s t+6 a$ & 98 & LBP 2663 \\
\hline & & T. venezuelensis & $\mathrm{F}$ & 52 & $20 \mathrm{~m}+16 \mathrm{sm}+16 \mathrm{st}$ & 104 & Nirchio et al. $2007 \mathrm{a}$ \\
\hline \multirow[t]{2}{*}{ Cyprinodontiformes } & Cyprinodontidae & Cyprinodon dearborni & M & 48 & $2 \mathrm{~m}+38 \mathrm{sm}+8 \mathrm{a}$ & 88 & Nirchio et al. $2003 \mathrm{a}$ \\
\hline & Rivulidae & Rivulus hartii & $\mathrm{F}$ & 44 & $2 m+4 s m+8 s t+30 a$ & 58 & Nirchio et al. $2005 \mathrm{~b}$ \\
\hline Elopiformes & Elopidae & Elops saurus & M & 50 & $6 \mathrm{~m}+4 \mathrm{t}+40 \mathrm{a}$ & 60 & LBP 6042 \\
\hline \multirow[t]{2}{*}{ Gymnotiformes } & Rhamphichthyidae & Rhamphichthys sp & $\mathrm{F}$ & 50 & $22 m+18 s m+6 s t+4 a$ & 96 & LBP 9949 \\
\hline & Sternopygidae & Eigenmannia $\mathrm{sp}$ & $\mathrm{F}$ & 38 & $4 m+2 s m+6 s t+26 a$ & 50 & LBP 2225 \\
\hline Lophiiformes & Antennariidae & Antennarius multiocelatus & M & 48 & $12 \mathrm{~m}+16 \mathrm{st}-20 \mathrm{a}$ & 76 & LBP 6087 \\
\hline \multirow[t]{25}{*}{ Mugiliformes } & Mugilidae & Agonostomus monticola & $\mathrm{F}$ & 48 & $2 \mathrm{st}+46 \mathrm{a}$ & 50 & Nirchio et al. $2008 \mathrm{~b}$ \\
\hline & & Mugil curema & M & 24 & $22 m+2 s m$ & 48 & Rossi et al. 2005 \\
\hline & & M. incilis & M & 48 & $48 \mathrm{a}$ & 48 & Het et al. 2011 \\
\hline & & M. liza & M & 48 & $48 \mathrm{a}$ & 48 & Rossi et al. 2005 \\
\hline & & M. rubrioculus & M & 48 & $48 \mathrm{a}$ & 48 & Nirchio et al. $2007 \mathrm{~b}$ \\
\hline & & Mugil sp. & M & 28 & $20 m+4 s t+4 a$ & 52 & LBP 6128 \\
\hline & & M. trichodon & M & 48 & $48 \mathrm{a}$ & 48 & Nirchio et al. 2005 a \\
\hline & Carangidae & Selene vomer & M & 46 & $2 \mathrm{st}+46 \mathrm{a}$ & 50 & LBP 6068 \\
\hline & & Trachinotus falcatus & M & 48 & $2 m+2 s t+44 a$ & 52 & LBP 6082 \\
\hline & Centropomidae & Centropomus undecimalis & M & 48 & $48 \mathrm{a}$ & 48 & LBP 6046 \\
\hline & Cichlidae & Cichla orinocensis & $\mathrm{F}$ & 48 & $48 \mathrm{a}$ & 48 & ECAM 387 \\
\hline & & Geophagus surinamensis & $\mathrm{F}$ & 48 & $2 \mathrm{~m}+2 \mathrm{sm}+12 \mathrm{st}+32 \mathrm{a}$ & 64 & ECAM 379 \\
\hline & Ephippidae & Chaetodipterus faber & M & 48 & $48 \mathrm{a}$ & 48 & Narváez 2000 \\
\hline & Gerreidae & Eucinostomus argenteus & M & 48 & $48 \mathrm{a}$ & 48 & LBP 6090 \\
\hline & Haemulidae & Haemulon aurolineatum & M & 48 & $48 \mathrm{a}$ & 48 & Nirchio et al. 2006a \\
\hline & & H. bonariensis & M & 48 & $48 \mathrm{a}$ & 48 & Nirchio et al. 2006a \\
\hline & & H. flavolineatum & M & 48 & $48 \mathrm{a}$ & 48 & Ron \& Nirchio 2005 \\
\hline & & H. plumierii & M & 48 & $48 \mathrm{a}$ & 48 & Nirchio et al. 2006 a \\
\hline & & H. sciurus & M & 48 & $48 \mathrm{a}$ & 48 & ECAM 1021 \\
\hline & & H. steindachneri & M & 46 & $46 a$ & 46 & ECAM 1022 \\
\hline & & H. striatum & M & 48 & $48 \mathrm{a}$ & 48 & ECAM 1019 \\
\hline & & Orthopristis ruber & M & 48 & $48 \mathrm{a}$ & 48 & ECAM 1017 \\
\hline & Labridae & Halichoeres bivittatus & M & 48 & $48 \mathrm{a}$ & 48 & LBP 6056 \\
\hline & & Lachnolaimus maximus & M & 48 & $6 m+16 s t+26 a$ & 70 & ECAM 1018 \\
\hline & & Xyrichthys novacula & M & 46 & $2 m+4 s m+40 a$ & 52 & ECAM 1031 \\
\hline
\end{tabular}


Table 2 (cont.). Karyotype characteristics for 103 species of fishes from Venezuela. $\mathrm{M}=$ marine, $\mathrm{F}=$ freshwater, $\mathrm{m}=$ meta-, $\mathrm{sm}$ $=$ submeta-, $\mathrm{st}=$ subtelo- and $\mathrm{a}=$ acrocentric chromosomes. In the last column the catalog numbers of the voucher specimens of species whose results are firstly presented here. Classification follows Eschmeyer \& Fong (2014).

\begin{tabular}{|c|c|c|c|c|c|c|c|}
\hline Order & Family & Species & Habitat & $2 n$ & Karyotype formulae & $\mathrm{FN}$ & $\begin{array}{l}\text { Reference or catalog } \\
\text { number of voucher }\end{array}$ \\
\hline \multirow[t]{19}{*}{ Perciformes } & \multirow[t]{6}{*}{ Lutjanidae } & Lutjanus analis & $\mathrm{M}$ & 48 & $48 \mathrm{a}$ & 48 & Nirchio et al. 2008 \\
\hline & & L. apodus & M & 48 & $48 \mathrm{a}$ & 48 & LBP 6052 \\
\hline & & L. griseus & M & 48 & $48 \mathrm{a}$ & 48 & Nirchio et al. 2008 \\
\hline & & L. synagris & M & $47 / 48$ & $1 \mathrm{~m}+46 \mathrm{a} / 48 \mathrm{a}$ & 48 & Nirchio et al. 2008 \\
\hline & & Ocyurus chrysurus & M & 48 & $48 \mathrm{a}$ & 48 & Nirchio et al. 2009 \\
\hline & & Rhomboplites aurorubens & M & 48 & $2 \mathrm{st}+46 \mathrm{a}$ & 50 & Nirchio et al. 2009 \\
\hline & Opistognathidae & $\begin{array}{l}\text { Opistognathus } \\
\text { macrognathus }\end{array}$ & M & 40 & $1 \mathrm{~m}+6 \mathrm{sm}+2 \mathrm{st}+28 \mathrm{a}$ & 46 & $\begin{array}{l}\text { Nirchio \& Oliveira } \\
2012\end{array}$ \\
\hline & \multirow[t]{2}{*}{ Pomacanthidae } & Pomacanthus arcuatus & M & 48 & $48 \mathrm{a}$ & 48 & LBP 6071 \\
\hline & & P. paru & M & 48 & $2 \mathrm{st}+46 \mathrm{a}$ & 50 & LBP 6070 \\
\hline & \multirow[t]{3}{*}{ Scaridae } & Nicholsina usta & M & 48 & $8 m+10 s m+6 s t+24 a$ & 72 & LBP 6054 \\
\hline & & Sparisoma aurofrenatum & M & 46 & $12 \mathrm{~m}+12 \mathrm{sm}+12 \mathrm{st}+10 \mathrm{a}$ & 82 & LBP 6055 \\
\hline & & S. chrysopterum & M & 46 & $6 \mathrm{~m}+12 \mathrm{sm}+10 \mathrm{st}+18 \mathrm{a}$ & 74 & LBP 6096 \\
\hline & \multirow[t]{4}{*}{ Scianidae } & Bairdiella ronchus & M & 48 & $48 \mathrm{a}$ & 48 & LBP 6436 \\
\hline & & B. sanctaluciae & M & 48 & $48 \mathrm{a}$ & 48 & LBP 6435 \\
\hline & & Ophioscion punctatissimus & M & 48 & $48 \mathrm{a}$ & 48 & LBP 6085 \\
\hline & & Stellifer sp. & M & 48 & $48 \mathrm{a}$ & 48 & LBP 6437 \\
\hline & \multirow[t]{2}{*}{ Serranidae } & Diplectrum formosum & M & 48 & $2 m+46 a$ & 50 & LBP 6093 \\
\hline & & Paralabrax dewegeri & M & 48 & $48 \mathrm{a}$ & 48 & LBP 6097 \\
\hline & Sparidae & Archosargus rhomboidalis & M & 48 & $14 m-s m+34 a$ & 62 & LBP 6439 \\
\hline \multirow[t]{2}{*}{ Pleuronectiformes } & \multirow[t]{2}{*}{ Paralichthyidae } & Citharichthys spilopterus & M & 26 & $14 m+4 s m+4 s t+4 a$ & 48 & ECAM 1016 \\
\hline & & Etropus crossotus & M & 36 & $11 \mathrm{~m}+14 \mathrm{st}+11 \mathrm{a}$ & 61 & LBP 6049 \\
\hline \multirow[t]{6}{*}{ Scorpaeniformes } & Dactylopteridae & Dactylopterus volitans & M & 48 & $14 m+14 s m+6 s t+14 a$ & 82 & ECAM 901 \\
\hline & \multirow[t]{4}{*}{ Scorpaenidae } & Pterois volitans & M & 48 & $2 \mathrm{~m}+8 \mathrm{sm}+8 \mathrm{st}+30 \mathrm{a}$ & 66 & LBP 13291 \\
\hline & & Scorpaena brasiliensis & M & 46 & $2 \mathrm{sm}+44 \mathrm{st}-\mathrm{a}$ & 48 & LBP 6044 \\
\hline & & S. isthmensis & M & 38 & $8 m+10 s t+20 a$ & 56 & LBP 6045 \\
\hline & & S. plumieri & M & 48 & $2 \mathrm{~m}+32 \mathrm{st}+14 \mathrm{a}$ & 82 & ECAM 1072 \\
\hline & Triglidae & Prionotus punctatus & M & 100 & $100 \mathrm{a}$ & 100 & ECAM 900 \\
\hline \multirow[t]{9}{*}{ Siluriformes } & \multirow[t]{2}{*}{ Ariidae } & Cathorops spixii & M & 52 & $14 \mathrm{~m}+20 \mathrm{sm}+18 \mathrm{st}$ & 104 & Nirchio et al. 2010 \\
\hline & & Sciades herzbergii & M & 54 & $14 \mathrm{~m}+20 \mathrm{sm}+18 \mathrm{st}+2 \mathrm{a}$ & 106 & Nirchio et al. 2010 \\
\hline & Callichthyidae & Hoplosternum littorale & $\mathrm{F}$ & 60 & $6 \mathrm{~m}+2 \mathrm{sm}+2 \mathrm{st}+50 \mathrm{a}$ & 70 & Nirchio et al. $2006 \mathrm{~b}$ \\
\hline & \multirow[t]{2}{*}{ Loricariidae } & Glyptoperichthys gibbiceps & $\mathrm{F}$ & 52 & $20 m+24 s m+8 s t$ & 104 & Alves et al. 2006 \\
\hline & & Liposarcus multiradiatus & $\mathrm{F}$ & 52 & $22 \mathrm{~m}+18 \mathrm{sm}+12 \mathrm{st}$ & 104 & Alves et al. 2006 \\
\hline & \multirow[t]{3}{*}{ Pimelodidae } & Pimelodus blockii & $\mathrm{F}$ & 56 & $18 \mathrm{~m}+16 \mathrm{sm}+10 \mathrm{st}+12 \mathrm{a}$ & 100 & LBP 3038 \\
\hline & & $\begin{array}{l}\text { Pseudoplatystoma } \\
\text { metaense }\end{array}$ & $\mathrm{F}$ & 56 & $24 m+18 s m+14 a$ & 98 & Nirchio et al. 2013 \\
\hline & & P. orinocoense & $\mathrm{F}$ & 56 & $24 m+18 s m+14 a$ & 98 & Nirchio et al., 2013 \\
\hline & Pseudopimelodidae & Cephalosilurus apurensis & $\mathrm{F}$ & 54 & $6 \mathrm{~m}+28 \mathrm{sm}+14 \mathrm{st}+6 \mathrm{a}$ & 102 & Martinez et al. 2008 \\
\hline \multirow[t]{3}{*}{ Synbranchiformes } & \multirow[t]{3}{*}{ Synbranchidae } & Ophisternon aenigmaticum & $\mathrm{F}$ & $45 / 46$ & $6 m+1 s t+38 a / 6 m+2 s t+38 a$ & 52 & Nirchio et al. 2011 \\
\hline & & & & & & 54 & \\
\hline & & Synbranchus marmoratus & $\mathrm{F}$ & 44 & $6 m+38 a$ & 50 & LBP 2227 \\
\hline \multirow[t]{3}{*}{ Tetraodontiformes } & Monachantidae & Stephanolepis setifer & M & $33 / 34$ & $1 \mathrm{~m}+32 \mathrm{a} / 34 \mathrm{a}$ & 34 & $\begin{array}{l}\text { Nirchio \& Oliveira, } \\
2007\end{array}$ \\
\hline & \multirow[t]{2}{*}{ Tetraodontidae } & Sphoeroides testudineus & M & 46 & $24 m+22 a$ & 70 & LBP 6059 \\
\hline & & S. greeleyi & M & 48 & $10 \mathrm{~m}+8 \mathrm{sm}+12 \mathrm{st}+16 \mathrm{a}$ & 76 & LBP 6058 \\
\hline
\end{tabular}

Scatter-plot of diploid number (Figs. 2a-2c) and C-Value data (Figs. 2b-2d) were taken from the Animal Genome Size Database (Gregory, 2012), versus the phylogenetic position of Actinopterygii families. Again an inversely proportional relationship with highly significant correlation coefficients was obtained both when all the 646 species were considered and when the probable 93 polyploid species were excluded.

\section{Discussion}

In freshwater fishes both the average number of chromosomes and the FN were higher than in marine fishes, and a general higher degree of cytogenetic diversification and karyotype variation is observed, compared to a more conserved cytogenetic pattern in marine fishes. Few decades ago the difference between karyotypes of freshwater and marine fishes was already observed and considered related to a more stable environment at sea as compared to inland waters, with some exceptions (Nikolsky, 1976). More recently, it has been reported that the differences in chromosome number and arm number between marine and freshwater fishes could be explained taking into account topographic barriers common to freshwater environments that would hamper the gene flow between populations, leading to fixation of macro-structural alterations in chromosomes. On the contrary, in the marine environment, the occurrence of large populations, the absence of well-defined geographical barriers, and the large capacity of dispersal would contribute to homogenize populations, reducing karyotype diversification (Molina, 2007). This is congruent with the idea that speciation rates are higher in freshwater than in marine 

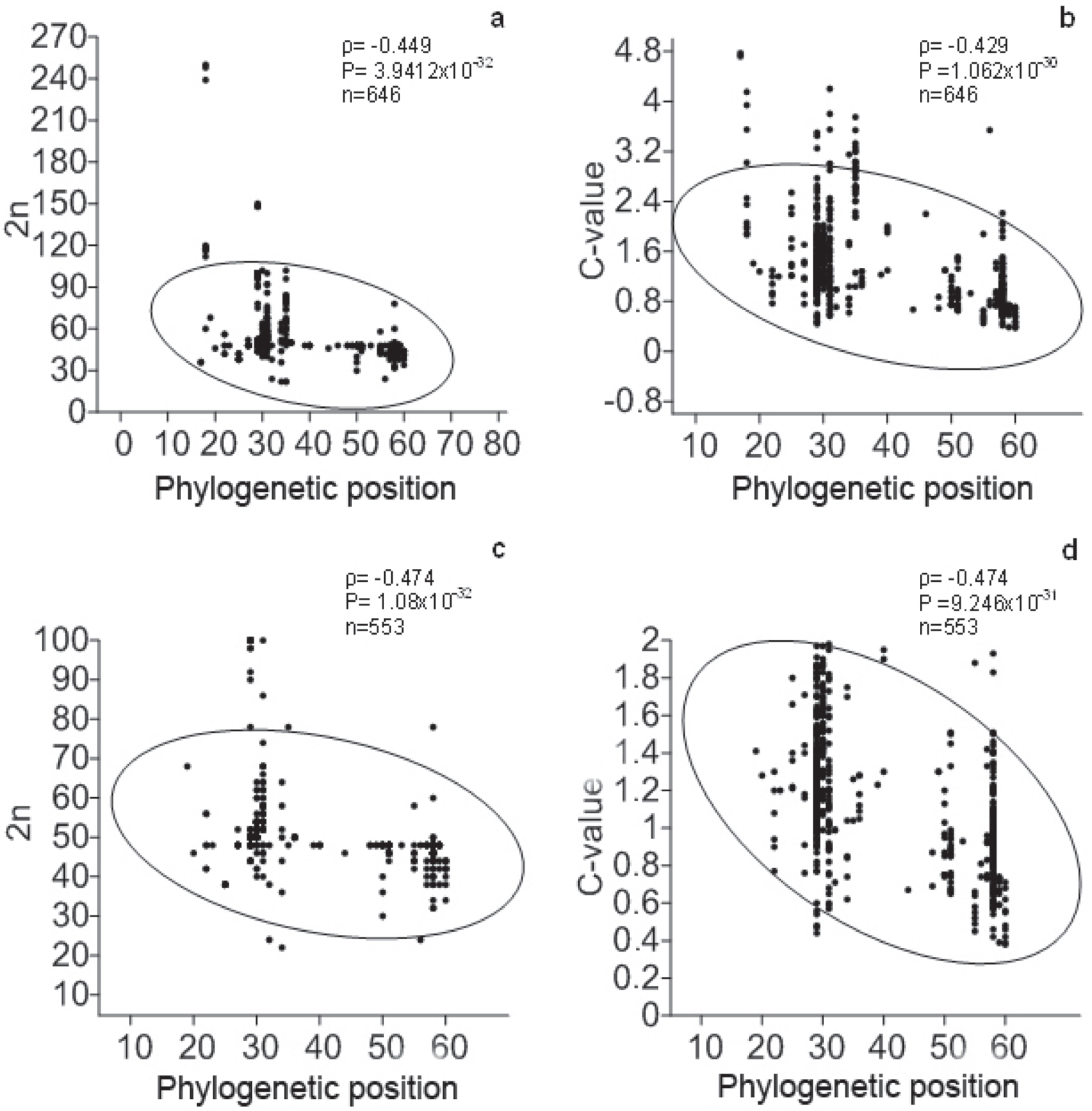

Fig. 2. Scatter-plot of (a, c) diploid number (2n), and (b, d) pg of DNA per haploid nucleus (C-Value), against the phylogenetic position of Actinopterygii families presented by Nelson (2006). Data include all available species (a, b) or exclude possible polyploidy species (c, d). Ellipses with $95 \%$ confidence are used as a correlation indicator.

fish lineages as the result of a greater number of barriers in the first habitat related to the latter (Bloom et al., 2013). The effects of mobility and population dimension on chromosome diversification seem to be confirmed when the analysis is limited to marine species that show different biological traits. Indeed marine taxa that have high mobility (eggs, larvae, or adults), like Haemulidae, Sciaenidae, Lutjanidae, and Serranidae (Perciformes) show 48 acrocentric chromosomes and reduced frequency of chromosomal macro-structural reorganizations.
On the contrary groups such as Muraenidae, Batrachoididae, and Scorpaenidae that do not form schools, are characterized by benthic habits, smaller populations, and more limited spatial locomotion, display a more extensive chromosomal diversity. However the apparent overall karyotype stability of Perciformes should be considered with caution, and could hide a more dynamic situation, i.e., the presence of microstructural rearrangements undetectable by conventional cytogenetic methods but visible after molecular banding, as reported over 
Table 3. Results of Chi-square $\left(\chi^{2}\right)$ test between freshwater and marine environment and between Perciformes and other Orders. $\mathrm{N}=$ Number of species.

\begin{tabular}{|c|c|c|c|c|c|c|}
\hline \multirow[b]{2}{*}{ Environment } & \multicolumn{2}{|c|}{ Diploid number } & \multirow[b]{2}{*}{$\mathrm{N}$} & \multirow[b]{2}{*}{$\chi^{2}$} & \multirow[b]{2}{*}{ D.F. } & \multirow[b]{2}{*}{$P$-value } \\
\hline & $2 n \neq 48$ & $2 n=48 A$ & & & & \\
\hline Freshwater & 32 & 1 & 33 & 126057 & & 0000 \\
\hline Marine & 45 & 25 & 70 & 12.6957 & 1 & 0.0004 \\
\hline $\begin{array}{ll} & \text { Total } \\
\text { Order } & \end{array}$ & 77 & 26 & 103 & & & \\
\hline Perciformes & 16 & 22 & 38 & 240003 & 1 & 000001 \\
\hline Other orders & 61 & 4 & 65 & 34.0203 & 1 & $<0.0001$ \\
\hline Total & 75 & 26 & 103 & & & \\
\hline
\end{tabular}

the last decade in marine fishes with conserved karyotypes like Serranidae (see references in Galetti et al., 2000), Haemulidae (Nirchio et al., 2006) and Lutjanidae (Nirchio et al., 2008) among others. This has also been observed in Mugiliformes, an order considered to be karyotypically conserved but in which recent studies carried out by the application of differential staining techniques and fluorescence in situ hybridization (FISH) with several DNA probes allowed the detection of a considerable number of microstructural changes among species (Sola et al., 2007,2008).

The highly significant probability (more than $99 \%$ confidence) of the Spearman correlation analysis, and the scatter-plot derived from our data clearly indicate that the most ancestral fishes, tend to have higher chromosome number compared to the more derived fishes (Figs. 1-2), and that a karyotype with $2 \mathrm{n}=48$ characterize the more derived groups, mainly Perciformes (Table 3 ). Moreover our estimates on the number of species belonging to this order showing a karyotype composed of 48 acrocentric chromosomes (57.89\%) is comparable to that $(60 \%)$ provided on a global level (Galetti et al., 2000).

The evidence presented herein challenge both the longlasting hypothesis that a karyotype composed of 48 acrocentric chromosomes could be ancestral for all teleost (Ohno, 1970), and the estimates of the chromosome number of the Actinopterygii proto-karyotype, dated 450 million, based on an analysis of the sequence of the genome of Tetraodon nigroviridis (Tetraodontiformes) (Jaillon et al., 2004). Furthermore present data do not agree with the indication of an ancestral karyotype probably composed of less than 48 chromosomal elements (Mank \& Avise, 2006), obtained mapping the number of chromosomes on the topology generated by molecular phylogenetic study for the Actinopterygii (Mank et al., 2005).

In fact, in the more basal Actinopterygii as the Polypteriformes and Acipenseriformes, the number of chromosomes is usually larger, ranging from 120-260 for Acipenseridae (Fontana et al., 1998) to 120 for Polyodontidae and 56-68 for Lepisosteidae (see references in Sola et al., 1981). Although extant Acipenseriformes families may have originated from a tetraploid ancestor with a probable karyotype of 120-macro and micro-chromosomes, it is presumed that the tetraploidization occurred at an early stage during the evolution of the group (Birstein et al., 1997) from an ancestor with 60 chromosomes (Dingerkus \& Howell, 1976; Carlson et al., 1982). On the other hand the presence of a karyotype close to $2 \mathrm{n}=60$ in the most ancient orders, is congruent with other studies. Indeed, the original idea that the ancestral karyotype of the Teleostei had approximately 60 elements and that the reduction in the number of chromosomes to 48 occurred independently in more than one phylogenetic line through fusions and deletions (Dingerkus, 1979) was proposed by Brum \& Galetti (1997). These authors suggested that the 48 chromosomes would be a synapomorphy extended among the more apical branches of the extant Teleostei (Clupeomorpha and Euteleostei) that was preserved mainly in marine species of Atherinomorpha and Percomorpha.

A general evolutionary tendency towards a reduction of chromosome number in fishes was also estimated by Nakatani et al. (2007) on the base of genome analysis suggesting that after two rounds of whole genome duplication (WGD), one occurred in the vertebrate proto-karyotype and one occurred in the Gnathostomata proto-karyotype, the number of chromosomes in the osteichthyan ancestor (after the divergence of Chondrichthyes, about 450 Mya) was $n \approx 31$. After the divergence of ray-finned and lobe-finned fishes, along the Actinopterygii lineage, chromosome fusions reduced this number to $n \approx 13$, in teleost ancestor about 350 Mya. A third round of whole genome duplication (3R-WGD) in the teleost ancestor doubled the number of chromosomes to $\mathrm{n} \approx 26$. Since then, the number of chromosomes in the teleost lineage has remained nearly unchanged. These hypotheses are largely congruent with the present results.

Our data indicated a trend towards simplification of the karyotype along the phylogeny of the Actinopterygii, both in terms of the chromosome number and in the genome size (DNA content/nucleus). Although remarkable modification in genome size were reported to be unconnected with chromosome number in ray-finned fish (Mank \& Avise, 2006), the assays of DNA content of over 200 teleost fishes, suggested a correlation between DNA content and evolution in this group (Hinegardner, 1968). That is, the more derived species of fishes have less DNA content per nucleus than ancestral ones, suggesting implicitly that evolution and specialization in teleosts had been accompanied by loss of DNA (Hinergardner, 1968), as also recently confirmed by measures of DNA bp content on a dataset from 1,043 species of Actinopterygii representing 190 families (Yi \& Streelman, 2005). As an example the haploid genome of Acipenser brevirostrum Lesuer, 1818 belonging to one of the most basal orders (Acipenseriformes), is 20 times greater than that found in Arothron meleagris (Anonymous, 1798) which belongs to Tetraodontiformes, the most derived teleost order. Molecular analysis suggests that this reduction is the consequence of loss of repetitive sequences and/or other non-coding DNA sequences (Neafsey \& Palumbi, 2003), including satellite DNA, ribosomal genes and transposable elements (TEs) (Biémont, 2008; Fedoroff, 2012).

Recognizing that several evolutionary events at the chromosomal level (polyploidization, fusions, fissions) have occurred in different lineages of ray-finned fishes, data here 
provided reveals a trend towards simplification of the karyotype along the phylogeny of the Actinopterygii from the more basal to the more derived lineages with a general conservation of chromosome numbers around $2 n=48$ acrocentric elements in the last ones. This different point of view challenges the currently accepted hypothesis, overall, based on oversimplification of chromosomal data, that a karyotype composed of 48 acrocentric chromosomes could be ancestral for all teleosts.

Although certainly limited by the number of species examined, data here reported represent also an initial reference database for Neotropical fishes from Venezuela that can be used for future comparative analyses aimed at better understanding the evolution of fishes, on the light of the huge expanding researches on genomes. Indeed, recent literature based on genetic maps and sequencing provided new evidence on debated aspects of genome evolution. For instances many authors argued that the 3R-WGD could be responsible for "species richness" in teleosts, but doubt about this direct association were raised (Santini et al., 2009; Sato \& Nishida, 2010). Moreover, although fishes were reported as having high rates of chromosomal rearrangements and low rates of synteny within vertebrates (Robinson-Rechavi et al., 2001a, 2001b; Venkatesh, 2003; Ravi \& Venkatesh, 2008), more recent investigation indicated both the presence of large syntenic blocks within Teleosts and between Teleosts and other vertebrates (Mazzuchelli et al., 2012). This indicates the stability of the genome of these fishes over long evolutionary time scales (Guyomard et al., 2012; Schartl et al., 2013). In addition a higher conserved gene order was observed in fishes than in mammals by genome comparison of three teleost species (fugu, Tetraodon, and medaka) with those of three mammalian lineages (human, mouse, and opossum), and authors reported these results as "largely due to a lower rate of interchromosomal rearrangements in the teleosts" (Kai et al., 2011).

Thus an effort is necessary to fill the gap between chromosome and genomic data on fishes, and to integrate cytogenetics and genomics in comparative and evolutionary studies.

\section{Acknowledgments}

We wish to acknowledge Luciana Sola, Markos Alexandrou and Julio Pérez for their criticisms. This work was supported by Consejo de Investigación, Universidad de Oriente, Venezuela, and by Fundação de Amparo à Pesquisa do Estado de São Paulo (FAPESP), Brazil and Conselho Nacional de Desenvolvimento Científico e Tecnológico (CNPq), Brazil, to CO.

\section{Literature Cited}

Alves, A. L., C. Oliveira, M. Nirchio, A. Granado \& F. Foresti. 2006. Karyotypic relationships among the tribes of Hypostominae (Siluriformes: Loricariidae) with description of XO sex chromosome system in a Neotropical fish species. Genetica, 128: 1-9-.
Arai, R. 2011. Fish Karyotypes: A Check List: Springer.

Biémont, C. 2008. Genome size evolution: Within-species variation in genome size. Heredity, 101: 297-298.

Birstein, V. J., R. Hanner \& R. DeSalle. 1997. Phylogeny of the Acipenseriformes: cytogenetic and molecular approaches. Environmental Biology of Fishes, 48: 127-155.

Booke, H. E. 1968. Cytotaxonomic studies of the Coregonine fishes of the Great Lakes, USA: DNA and karyotype analysis. Journal of the Fishery Research Board of Canada, 25: 1667-1687.

Bloom, D. D., J. T. Weir, K. R. Piller \& N. R. Lovejoy. 2013. Do freshwater fishes diversify faster than marine fishes? A test using state-dependent diversification analyses and molecular phylogenetics of new world silversides (Atherinopsidae). Evolution, 67: 2040-2057.

Brum, M. J. I. \& P. M. Galetti. 1997. Teleostei ground plan karyotype. Journal of Computational Biology, 2: 91-102.

Carlson, D. M., M. K. Kettler, S. E. Fisher \& G. S. Whitt. 1982. Low genetic variability in paddlefish populations. Copeia, 1982: 721-725.

Clem, W. L., L. Moewus \& S. M. Siegel. 1961. Studies with cells from marine fish in tissue culture. Proceedings of the Society of Experimental Biology and Medicine, 108: 762-766.

Denton, T. E. 1973. Fish chromosome methodology. Illinois: Charles C. Thomas Publisher.

Dingerkus, G. \& W. M. Howell. 1976. Karyotypic analysis and evidence of tetraploidy in the North American paddlefish, Polyodon spathula. Science, 177: 664-669.

Dingerkus, G. 1979. Chordate cytogenetic studies: An analysis of their phylogenetic implications with particular reference to fishes and the living coelacanth. Occasional Paper of the California Academy of Science, 134: 111-127.

Eschmeyer, W. N. \& J. D. Fong. 2014. Species by Family/Subfamily. Available at http://research.calacademy.org/research/ ichthyology/catalog/SpeciesByFamily.asp (July 2014)

Fedoroff, N. V. 2012. Transposable elements, epigenetics, and genome evolution. Science, 338: 758-767.

Fontana, F., J. Tagliavini, L. Congiu, M. Lanfredi, M. Chicca, C. Laurente \& R. Rossi. 1998. Karyotypic characterization of the great sturgeon, Huso huso, by multiple staining techniques and fluorescent in situ hybridization. Marine Biology, 132: 495-501.

Froese, R. \& D. Pauly (Eds.). 2013. FishBase. World Wide Web electronic publication. Available at http:// www.fishbase.org,version 04/2013 (May 2013).

Galetti, P. M. Jr., C. T. Aguilar \& W. F. Molina. 2000. An overview of marine fish cytogenetics. Hydrobiologia, 420: 55-62.

Gaviria, J. I., M. Nirchio, A. Granado \& A. Estrada. 2005. Karyotype and nucleolar organizer regions of Pygocentrus cariba (Humboldt, 1821) (Pisces: Serrasalminae) from Caicara del Orinoco, Venezuela. Interciencia, 30: 44-4.

Gregory, T. R. 2012. Animal Genome Size Database. Available at http://www.genomesize.com.

Guerra, M. 2012. Cytotaxonomy: The end of childhood. Plant Biosystems, 146: 703-710.

Guyomard, R., M. Boussaha, F. Krieg, C. Hervet \& E. Quillet. 2012. A synthetic rainbow trout linkage map provides new insights into the salmonid whole genome duplication and the conservation of synteny among teleosts. BMC Genetics, 13: 15.

Hammer, Ø., D. A. T. Harper \& P. D. Ryan. 2001. PAST: Paleontological Statistics Software Package for Education and Data Analysis. Palaeontologia Electronica, 4. Available at http:/ /folk.uio.no/ohammer/past/.

Hett, A. K., M. Nirchio, C. Oliveira, Z. R. Siccha, A. R. Rossi \& L. 
Sola. 2011. Karyotype characterization of Mugil incilis Hancock, 1830 (Mugiliformes: Mugilidae), including a description of an unusual co-localization of major and minor ribosomal genes in the family. Neotropical Ichthyology, 9: 107-112.

Hinegardner, R. 1968. Evolution of Cellular DNA Content in Teleost Fishes. American Naturalist, 102: 517-523.

Jaillon, O., J-M. Aury, F. Brunet, J-L. Petit, N. Stange-Thomann, E. Mauceli, L. Bouneau, C. Fischer, C. Ozouf-Costaz, A. Bernot, S. Nicaud, D. Jaffe, S. Fisher, G. Lutfalla, C. Dossat, B. Segurens, C. Dasilva, M. Salanoubat, . Levy, N. Boudet, S. Castellano, V. Anthouard, C. Jubin, V. Castelli, M. Katinka, B. Vacherie, C. Biemont, Z. Skalli, L. Cattolico, J. Poulain, V. de Berardinis, C. Cruaud, S. Duprat, P. Brottier, J-P. Coutanceau, J. Gouzy, G. Parra, G. Lardier, C. Chapple, K.J. McKernan, P. McEwan, S. Bosak, M. Kellis, J-N. Volff, R. Guigo, M.C. Zody, J. Mesirov, K. Lindblad-Toh, B. Birren, C. Nusbaum, D. Kahn, M. Robinson-Rechavi, V. Laudet, V. Schachter, F. Quetier, W. Saurin, C. Scarpelli, P. Wincker, E. Lander, J. Weissenbach \& H.R. Crollius. 2004. Genome duplication in the teleost fish Tetraodon nigroviridis reveals the early vertebrate proto-karyotype. Nature, 431: 946-957.

Kai, W., K. Kikuchi, S. Tohari, A. K. Chew, A. Tay, A. Fujiwara, S. Hosoya, H. Suetake, K. Naruse, S. Brenner, Y. Suzuki \& B. Venkatesh. 2011. Integration of the genetic map and genome assembly of fugu facilitates insights into distinct features of genome evolution in teleosts and mammals. Genome Biology and Evolution, 3: 424-442.

Klinkhardt, M., M. Tesche \& H. Greven. 1995. Database of fish chromosomes. Germany: Westarp Wissenschaften, Magdeburg.

Levan, A., D. Fredga \& A. Sandberg. 1964. Nomenclature for centromeric position on chromosomes. Hereditas, 52: 201-220.

Mank, J. E. \& J. C. Avise. 2006. Phylogenetic conservation of chromosome numbers in Actinopterygiian fishes. Genetica, 127: 321-327.

Mank, J. E., D. E. L. Promislow \& J. C. Avise. 2005. Phylogenetic perspectives on the evolution of parental care in fishes. Evolution, 59: 1570-1578.

Mariguela, T. C., M. Nirchio, E. Ron, J. I. Gaviria, F. Foresti \& C. Oliveira. 2010. Cytogenetic characterization of Brycon amazonicus (Spix et Agassiz, 1829) (Teleostei: Characidae) from Caicara del Orinoco, Venezuela. Comparative Cytogenetics, 4: 185-193.

Martinez, E. R. M., M. Nirchio, A. Granado, F. Foresti \& C. Oliveira. 2008. Cytogenetic analysis of three catfish species of the family Pseudopimelodidae (Teleostei: Siluriformes). Genetics and Molecular Biology, 31: 692-696.

Mazzuchelli, J., T. D. Kocher, F. Yang \& C. Martins. 2012. Integrating cytogenetics and genomics in comparative evolutionary studies of cichlid fish. BMC Genomics, 13: 463.

Miloslavich, P., E. Klein, E. Yerena \& A. Martin. 2003. Marine biodiversity in Venezuela: status and perspectives. Gayana (Concepc.), 67: 275-301.

Molina, W. F. 2007. Chromosomal changes and stasis in marine fish groups. Pp. 69-110. In: Pisano, E., C. Ozouf-Costaz, F. Foresti, B.G. Kapoor (Eds.). Fish Cytogenetics. Enfield, NH: Science Publishers.

Nakatani, Y., H. Takeda, Y. Kohara \& S. Morishita. 2007. Reconstruction of the vertebrate ancestral genome reveals dynamic genome reorganization in early vertebrates. Genome Research, 17: 1254-1265

Narváez, L. 2000. Variación genética por electroforesis y cariotipo de Chaetodipterus faber. Unpublished Thesis, Universidad de
Oriente, Núcleo Nueva Esparta, Isla de Margarita, Venezuela, $68 \mathrm{p}$.

Neafsey, D. E. \& S. R. Palumbi. 2003. Genome size evolution in pufferfish: a comparative analysis of diodontid and tetraodontid pufferfish genomes. Genome Research, 13: 821-839.

Nelson, S. J. 2006. Fishes of the World, $4^{\text {th }}$ Ed., New York: John Wiley and Sons Inc.

Nikolsky, G. 1976. The Interrelation between variability of characters, effectiveness of energy utilization, and karyotype structure in fishes. Evolution, 30: 180-185.

Nirchio, M. \& C. Oliveira. 2006. Citogenética de Peces. Cumaná, Venezuela, Universidad de Oriente.

Nirchio, M. \& C. Oliveira. 2007. First description of the karyotype and Ag-NORs localization of Stephanolepis setifer (Bennett, 1831) (Tetraodontiformes: Monacanthidae) with remarks on sex chromosome differentiation. Boletin del Instituto Oceanografico de Venezuela Universidad de Oriente, 46: 117-123.

Nirchio, M. \& C. Oliveira. 2012. Classical cytogenetic characterization of Opistognathus macrognathus (Perciformes: Opistognathidae). Boletin del Instituto Oceanografico de Venezuela Universidad de Oriente, 51: 123-127.

Nirchio, M., B. J. Turner, J. E. Pérez, J. I. Gaviria \& H. Cequea. 2001. Karyotypes of three species of toadfish (Batrachoididae: Teleostei) from Margarita Island, Venezuela. Scientia Marina, 66: 3-4.

Nirchio, M., A. Granado, E. Ron \& J. E. Pérez. 2002. Karyotype and Nucleolar Organizer Regions in Serrasalmus rhombeus (Linnaeus, 1766) (Serrasalminae) from Caicara Del Orinoco, Venezuela. Interciencia, 27: 676-678.

Nirchio, M., H. Cequea, B. J. \& B. J. Turner, B. J. 2003 a. Karyotypic characterization and nucleolus organizer regions in Cyprinodon dearborni (Meek, 1909) (Pisces: Cyprinodontidae) from Venezuela. Interciencia, 28: 1-4.

Nirchio, M., A. S. Fenocchio, A. C. Swarça, J. E. Pérez, A. Granado, A. Estrada \& E. Ron. 2003 b. Cytogenetic characterization of hybrids offspring between Colossoma macropomum (Cuvier, 1818) and Piaractus brachypomus (Cuvier, 1817) from Caicara del Orinoco, Venezuela. Caryologia, 56: 405-411.

Nirchio, M., A. S. Fenocchio, A. C. Swarça, A. L. Dias, L. GiulianoCaetano, E. Ron, J. I. Gaviria \& J. E. Pérez. 2004a. Cytogenetic characterization of Thalassophryne maculosa Günther, 1861 (Pisces: Batrachoididae) from Margarita Island, Venezuela. Caribbean Journal of Science, 40: 218-222.

Nirchio, M., A. S. Fenocchio, A. C. Swarça \& J. E. Pérez. 2004b. Karyology of the toadfish Porichthys plectrodon (Jordan and Gilbert, 1882) (Batrachoididae) from Margarita Island, Venezuela. Marine Biology, 146: 161-165.

Nirchio, M., E. Ron \& A. R. Rossi. 2005a. Karyological characterization of Mugil trichodon Poey, 1876 (Pisces: Mugilidae) from Margarita Island, Venezuela. Scientia Marina, 69: 525-530.

Nirchio, M., J. A. Gómez, C. Oliveira, E. Ron, M. Nirchio Jr. \& J. Villaroel. 2005b. Rivulus hartii (Boulenger, 1890) (Cyprinodontiformes: Rivulidae): Especie de pez dulceacuícola amenazada en la Isla de Margarita, Venezuela. Tecnociencia, 7: 135-142.

Nirchio, M., J. I. Gaviria, C. Oliveira, A. I. Ferreira \& C. Martins. 2006a. Cytogenetic analysis of three species of the genus Haemulon (Teleostei: Haemulinae) from Margarita island, Venezuela. Genetica, 131: 135-140.

Nirchio, M., J. E. Pérez, A. Granado \& E. Ron. 2006 b. Conventional karyotype, constitutive heterocromatin, and nucleolar organizer 
regions, in Hoplosternum littorale (Pisces: Callichthyidae) from Caicara del Orinoco, Venezuela. Saber, Universidad de Oriente, Venezuela, 18: 113-116.

Nirchio, M., C. Oliveira, A. I. Ferreira, A. Granado \& E. Ron. 2007a. Extensive polymorphism and chromosomal characteristics of ribosomal DNA in the characid fish Triportheus venezuelensis (Characiformes, Characidae). Genetetics and Molecular Biology, 30: 25-30.

Nirchio, M., C. Oliveira, A. I. Ferreira, J. E. Pérez, J. I. Gaviria, I. Harrison, A. R. Rossi \& L. Sola. 2007b. Comparative cytogenetic and allozyme analysis of Mugil rubrioculus and M. curema (Teleostei, Mugilidae) from Venezuela. Interciencia, 32: 757-762.

Nirchio, M., R. Rondon, J. E. Pérez, C. Oliveira, A. I. Ferreira, C. Martins, A. R. Rossi \& L. Sola. 2008a. Cytogenetic studies in three species of Lutjaninae (Teleostei) from Margarita Island, Venezuela. Neotropical Ichthyology, 6: 101-108.

Nirchio, M., C. Oliveira, A. I. Ferreira, C. Martins, A. R. \& L. Sola. 2008b. Classical and molecular cytogenetic characterization of Agonostomus monticola, a primitive species of Mugilidae (Mugiliformes). Genetica, 135: 1-5.

Nirchio, M., C. Oliveira, D. C. Ferreira,R. Rondón, J. E. Pérez, A. K. Hett, A. R. Rossi, \& L. Sola.2009. Cytogenetic characterization of Rhomboplites aurorubens and Ocyurus chrysurus, two monotypic genera of Lutjaninae from Cubagua Island, Venezuela, with a review of the cytogenetics of Lutjanidae (Teleostei: Perciformes). Neotropical Ichthyology, 7: 587-594.

Nirchio, M., E. R. Martinez, F. Foresti \& C. Oliveira. 2010. Cytogenetic analysis of three sea catfish species (Teleostei, Siluriformes, Ariidae) with the first report of Ag-NOR in this fish family. Genetics and Molecular Biology, 33: 262-265.

Nirchio, M., T. C. Mariguela, I. A. Ferreira, F. Foresti \& C. Oliveira C. 2011. Karyotype and nucleolus organizer regions of Ophisternon aenigmaticum (Teleostei: Synbranchiformes: Synbranchidae) from Venezuela. Interciencia, 36: 229-233.

Nirchio, M., A. Mujica, C. Oliveira, A. Granado, J. Mora, A. K. Hett, A. R. Rossi, V. Milana, L. Sola. 2013. Pseudoplatystoma metaense and P. orinocoense (Siluriformes: Pimelodidae) from the Orinoco basin, Venezuela: cytogenetic and molecular analyses. Italian Journal of Zoology, 80 (4): 526-535.

Ohno, S. 1970. Evolution by gene duplication. New York: Springer Verlag.

Ojima, Y., K. Ueno \& M. Hayashi. 1976. A review of the chromosome numbers in fishes. La Kromosomo, 1: 19-47.

Oliveira, C., L. F. Almeida-Toledo, F. Foresti, H. A. Britski \& A. S. Toledo-Filho. 1988. Chromosome formulae of Neotropical freshwater fishes. Revista Brasilera de Genetica, 11: 577-624.

Oliveira, C., M. Nirchio, A. Granado \& S. Levy. 2003. Karyotypic characterization of Prochilodus mariae, Semaprochilodus kneri and S. laticeps (Teleostei: Prochilodontidae) from Caicara del Orinoco, Venezuela. Neotropical Ichthyology, 1: 47-52.

Ozouf-Costaz, C. \& F. Foresti. 1992. Fish cytogenetic research: advances, applications and perspectives. Netherland Journal of Zoology, 42: 277-290.

Park, E. H. 1974. A list of the chromosome numbers of finfishes. College Review. College of Liberal Arts and Sciences Seoul National University, 20: 346-372.

Pisano, E., C. Ozouf-Costaz, F. Foresti \& B. G. Kapoor. 2007. (Eds.). Fish Cytogenetics. Enfield N. H., Science Publishers Inc. (USA).

Porto, J. I. R., E. Feldberg, C. M. Nakayama \& J. N. Falcao. 1992. A checklist of chromosome numbers and karyotypes of Amazonian freshwater Fishes. Revue d' Hydrobiologie Tropicale, 25: 287-299.
Ravi, V. \& B. Venkatesh. 2008. Rapidly evolving fish genomes and teleost diversity. Current Opinion in Genetics and Development, 18: 544-550.

Robinson-Rechavi, M., O. Marchand, H. Escriva, P. Bardet, D. Zelus, S. Hughes \& V. Laudet. 2001. Euteleost fish genomes are characterized by expansion of gene families. Genome Reseach, 11: 781-788.

Robinson-Rechavi, M., O. Marchand, H. Escriva \& V. Laude. 2001. An ancient whole genome duplication may not have been responsible for the abundance of duplicated fish genes. Current Biology, 11: 458-459.

Ron, E. \& M. Nirchio. 2005. Caracterización Citogenética de Haemulon flavolineatum Desmarest, 1823 (Pisces: Haemulidae) de la Isla de Margarita, Venezuela. Boletin del Instituto Oceanográfico de Venezuela Universidad de Oriente, 44: 35-40.

Rossi, A. R., E. Gornung, L. Sola \& M. Nirchio. 2005. Comparative molecular cytogenetic analysis of two congeneric species, Mugil curema and M. liza (Pisces, Mugiliformes), characterized by significant karyotype diversity. Genetica, 125: 27-32.

Santini, F., L. J. Harmon, G. Carnevale \& M. E. Alfaro. 2009. Did genome duplication drive the origin of teleosts? A comparative study of diversification in ray-finned fishes. BMC Evolutionary Biology, 9: 194.

Sato, Y. \& M. Nishida. 2010. Teleost fish with specific genome duplication as unique models of vertebrate evolution. Environmental Biology of Fishes, 88: 169-188.

Schartl, M., R. B. Walter, Y. Shen, T. Garcia, J. Catchen, A. Amores, I. Braasch, D. Chalopin, J. N. Volff, K. P. Lesch, A. Bisazza, P. Minx, L. D. Hillier, R. K. Wilson, S. Fuerstenberg, J. Boore, S. Searle, J. H. Postlethwait \& W. C. Warren. 2013. The genome of the platyfish, Xiphophorus maculatus, provides insights into evolutionary adaptation and several complex traits. Nature Genetics, 45: 567-572.

Smith, E. M. \& T. R. Gregory. 2009. Patterns of genome size diversity in the ray-finned fishes. Hydrobiologia, 625: 1-25.

Sokal, R. R. \& F. J. Rohlf. 1995. Biometry: the principles and practice of statistics in biological research. New York: W.H. Freeman.

Sola, L., S. Cataudella \& E. Capanna. 1981. New developments in vertebrate cytotaxonomy. III. Karyology of bony fishes: a review. Genetica, 54: 285-328.

Sola, L., E. Gornung, M. E. Mannarelli \& A. R. Rossi. 2007. Chromosomal evolution in Mugilidae, Mugilomorpha: an overview. Pp. 165-194. In: Fish Cytogenetics. Pisano, E., C. Ozouf-Costaz, F. Foresti, B.G. Kapoor (Eds.). Enfield, NH: Science Publishers.

Sola, L., M. Nirchio \& A. R. Rossi. 2008. The Past and future of cytogenetics of mugilidae: an updated overview. Boletin del Instituto Oceanografico de Venezuela, Universidad de Oriente, 47: 25-32.

Vasiliev, V. P. 1985. Evolutionary karyology of fishes. Moscow: Publishing House Nauka.

Venkatesh, B. 2003. Evolution and diversity of fish genomes. Current Opinions in Genetics and Development, 13: 588-592.

WinSTAT. Available at http://www.winstat.com/

Wolf, K. \& M. C. Quimby. 1969. Fish cell and tissue culture. Pp. 253-305. In: W. S. Hoar \& D. J. Randall (Eds.). Fish Physiology. Academic Press, New York.

Yi, S. \& T. Streelman. 2005. Genome size is negatively correlated with effective population size in ray-finned fish. Trends in Genetics, 21: 643-646.

Submitted January 30, 2014

Accepted March 23, 2014 by Alexandre Hilsdorf Published December 27, 2014 